\title{
Frequency of Sexual Recombination by Mycosphaerella graminicola in Mild and Severe Epidemics
}

\author{
C. Cowger, P. C. Brunner, and C. C. Mundt
}

First author: U.S. Department of Agriculture-Agricultural Research Service, Department of Plant Pathology, North Carolina State University, Raleigh 27695; second author: ETH Zürich, Institute of Integrative Biology (IBZ); and third author: Department of Plant Pathology, Oregon State University, Cordley Hall 2082, Corvallis 97331.

Accepted for publication 26 February 2008.

\begin{abstract}
Cowger, C., Brunner, P. C., and Mundt, C. C. 2008. Frequency of sexual recombination by Mycosphaerella graminicola in mild and severe epidemics. Phytopathology 98:752-759.

The importance of sexual recombination in determining fungal population structure cannot be inferred solely from the relative abundance of sexual and asexual spores and reproductive structures. To complement a previously reported study of proportions of Mycosphaerella graminicola ascocarps and pycnidia, we investigated the share of sexual recombinants among isolates randomly derived from the same field at the same time. Early in three successive growing seasons (those ending in 1998, 1999, and 2000), field plots of the susceptible winter wheat cultivar Stephens were inoculated with suspensions of two $M$. graminicola isolates that each had rare alleles at restriction fragment length polymorphism (RFLP)

compare allele frequencies. Based on RFLP haplotypes and DNA fingerprints, isolates from the inoculated plots were categorized by both inspection and Bayesian methods as inoculant clones, recombinants, or immigrants. Inoculation in the 2000 season was delayed, and the recovery rate of inoculant types was just 1 to $2 \%$. In 1998, a high-disease year, and 1999, a low-disease year, inoculants comprised 36 and 22 to $23 \%$ of endof-season samples, respectively. In those 2 years, recombinants as a percentage of inoculant descendants (both sexual and asexual) were 35 and $32 \%$, respectively. By comparison, the study of fruiting bodies had found 93 and 32\% of M. graminicola fruiting bodies were ascocarps in 1998 and 1999, respectively. These findings support the hypothesis that sexual recombination makes a relatively consistent contribution to $M$. graminicola population structure, despite differences in epidemic severity and ascocarp proportions.
\end{abstract} loci. Near harvest time, leaves were randomly sampled from the same plots, and a population of over 100 monopycnidial isolates was created for each year of the experiment. Natural populations were also sampled from noninoculated plots in the 1999 and 2000 seasons, in order to
Additional keywords: mark-release-recapture, Septoria tritici, Septoria tritici blotch, Triticum aestivum.
For plant pathogens that reproduce both sexually and asexually, the influence of recombination on population structure may remain uncertain even when it is clear that a teleomorph exists and sexual propagules initiate epidemics. One must still determine the actual balance of sexual and asexual descendants in order to estimate the magnitude of sex within total reproduction and the influence of recombination on the pathogen's capacity to adapt to stresses such as host resistance or fungicides (14). In turn, the proportion of reproduction that is sexual may be influenced by factors that determine epidemic intensity, such as weather and host resistance $(5,8)$.

Among plant pathogens, this issue has perhaps been best studied using the Mycosphaerella graminicola-wheat (Triticum aestivum) pathosystem. Zhan et al. (23) inoculated $10 \mathrm{M}$. graminicola isolates with rare restriction fragment length polymorphism (RFLP) alleles onto plots of winter wheat in 1994. They used RFLP allele frequencies and DNA fingerprints to estimate that $24 \%$ of isolates sampled at the end of the season were sexual recombinants, a figure that they later revised slightly downward to $21 \%(2,24)$. However, the environment in a particular season is likely to have a significant influence on the amount of sexual reproduction in this pathosystem. Eriksen et al. (8) used a mathe-

Corresponding author: C. Cowger; E-mail address: christina.cowger@ars.usda.gov

doi:10.1094/PHYTO-98-7-0752

This article is in the public domain and not copyrightable. It may be freely reprinted with customary crediting of the source. The American Phytopathological Society, 2008. matical model to predict that the end-of-season share of $M$. graminicola sexual descendants would vary between 12 and $27 \%$. The higher percentages were attained if the simulation included a period of dry weather, as pycnidiospores require rain for dispersal and ascospores do not.

Cowger et al. (5) concluded from field evidence that the frequency of sexual reproduction in $M$. graminicola is positively associated with epidemic intensity and host susceptibility, which is logical as the pathogen is heterothallic and opposite mating types must come in contact for sex to occur (11). Averaged across multiple cultivars, the end-of-season percentage of identifiable $M$. graminicola fruiting bodies that were ascocarps varied from $63 \%$ in a high-disease year (1998) to $14 \%$ in a low-disease year (1999). The percentages of ascocarps on the susceptible cultivar Stephens were even more divergent: $93 \%$ in 1998 and 32\% in 1999. From these data alone, however, the authors could not infer the proportion of isolates at the end of each season that was the product of sexual recombination. Comparisons between years were also complicated by weather. For example, the observed share of $M$. graminicola fruiting bodies that were pycnidia could be reduced by rain and spore release in the presampling period, as empty fruiting bodies were not identified to species.

The number of $M$. graminicola sexual cycles that may occur during a single growing season is also unclear, with Kema et al. (11) suggesting the possibility of multiple sexual cycles per season, and others estimating that a maximum of one or two cycles is likely $(5,8)$. This would clearly affect the degree to which sexual recombination structures the population, and raises the question whether results depend on what time of year samp- 
ling is conducted. While there is broad agreement that M. graminicola ascospores initiate epidemics in fall-sown wheat $(10,11$, 20,21 ), the potential role of ascospores in subsequent disease development is less clear. Hunter et al. (10) found that M. graminicola ascospores could be trapped throughout the year at Long Ashton, UK, with high year-to-year variability in the month of greatest spore abundance, and they suggested ascospores released from the growing wheat crop could play a role in within-season disease development. However, DiLeone et al. (7) found that trap plants removed from an Oregon field at weekly intervals from early October to late April of the 1992 to 1993 growing season developed M. graminicola lesions almost exclusively in October and November. The Oregon fruiting body survey by Cowger et al. (5) suggested that ascocarps were absent until late May or early June, and then increased in frequency through the end of June, consistent with the hypothesis of only one or two annual sexual cycles and little or no role for ascospores after epidemic initiation.

Since Zhan et al. $(23,24)$ estimated $M$. graminicola recombination and immigration, Bayesian assignment methods have come into growing use in population genetics $(1,13)$. These powerful techniques now allow probabilistic assignment of individuals to populations, such as inference of migrant or recombinant ancestry, on the basis of multilocus genotypes and allele frequencies in reference populations.

The present study complemented that reported by Cowger et al. (5) above, and was designed to relate end-of-season proportions of sexual and asexual fruiting bodies to proportions of sexual and asexual descendants, using the mark-release-recapture approach of Zhan et al. (24). In the same field from which fruiting bodies were sampled, and using the same susceptible winter wheat cultivar Stephens, two M. graminicola isolates with known rare RFLP alleles were inoculated onto field plots near the beginning of each growing season for three successive years. At the end of each season, leaves were collected at random and isolates were derived from them. Isolates were identified as inoculant types, products of sexual recombination, or immigrants, based on their RFLP genotypes. Disease progress was assessed in order to relate epidemic intensities in the 3 years to observed sexual recombination frequencies. A small number of isolates from a moderately resistant cultivar were also evaluated in 1999 and 2000.

\section{MATERIALS AND METHODS}

Field plot establishment. In each of three successive years, field plots of the cultivar Stephens, which is susceptible to $M$. graminicola, were established at the Oregon State University Botany and Plant Pathology Field Laboratory in Corvallis, Oregon. The plots were part of a larger experiment with multiple research objectives that had a randomized complete block design with four replicate blocks. For purposes of the present study, in each year each replicate included one plot of Stephens used for inoculation and sampling. In the second and third years, each replicate also included a plot of the cultivar Foote, which is moderately resistant to $M$. graminicola and was inoculated, and a separate plot of Stephens that was not inoculated and was sampled separately. Inoculation and sampling are described below.

In the first 2 years, each plot consisted of two adjacent $1.5 \times$ $6.1 \mathrm{~m}$ planting units, while in the third year plots were only single planting units in order to conserve space. In each year, equalsized plots of wheat and barley were alternated in a checkerboard pattern so that the barley provided a buffer between the wheat plots. Plots were planted on 20 October 1997, 7 October 1998, and 12 October 1999. Fertilization, planting, and weed control were all conducted according to standard commercial practices in the area.

Inoculation and disease assessment. Two $M$. graminicola isolates that had originally been collected in Corvallis were kindly supplied by B. McDonald and were coinoculated in the field: a15-4A.9 and $\alpha 2-2$ A.12 (hereafter referred to as isolates 9 and 12). Both isolates had RFLP alleles at two or three loci that appeared at frequencies of less than $10 \%$ in natural Oregon populations of M. graminicola (Tables 1 and 2; B. McDonald, unpublished data). Isolate $\alpha 2-2 \mathrm{~A} .12$ was of mating type 1 and isolate a15-4A.9 was of mating type 2 (G. Kema, personal communication).

Field inoculations were performed on 19 November 1997, 12 November 1998, and 20 December 1999, with the later date in the third year owing to difficulties in producing inoculum. Inoculant isolates were grown in yeast-malt-sucrose broth amended with streptomycin at $50 \mathrm{mg} / \mathrm{liter}$. The spores were filtered through eight layers of cheesecloth, and concentrations were adjusted using a hemacytometer to make a suspension of $5 \times 10^{5}$ spores $/ \mathrm{ml}$ that was comprised of equal proportions of the two isolates. One drop of Tween was added per liter of spore suspension, and 1 liter of suspension was applied to each plot using a backpack sprayer. Plots were covered with tarps for 3 to 4 days following inoculation to encourage infection. Visual assessments of percent diseased leaf area were conducted by two assessors for each plot on a whole-canopy basis eight times each year between earlyFebruary and mid-June.

Leaf sampling. Leaf collections were made in all 3 years in inoculated Stephens plots. In the second and third years, leaf samples were also gathered in inoculated Foote plots, and in noninoculated Stephens plots, where natural populations of M. graminicola had established themselves.

Leaves with $M$. graminicola lesions were collected near the end of each growing season on 15 June 1998, 29 June 1999, and 27 June 2000. In 1998, a diagonal transect was established across each plot, and 10 flag leaves were collected blindly at the points where the transect crossed each of 10 rows. In other words, 10 tillers were sampled at random at each of 10 points approximately $0.67 \mathrm{~m}$ apart on a diagonal line across each plot. Leaves were not collected from the half-meter at each plot end, nor from the outermost row on each side, in order to minimize edge effects. In 1999, F-1 leaves were collected rather than flag leaves due to the scarcity of M. graminicola lesions on flag leaves. Sampling was carried out as in the first year, except that five leaves were sampled from each row instead of 10 . In 2000, six F-1 leaves were collected from each row, and the transect in each plot was in the shape of an $X$, because plots were half the width of the previous 2 years. The result in 2000 was that within a given plot, the distances between sampling points in adjacent rows ranged from approximately 0.3 to $2 \mathrm{~m}$. Sampling was otherwise as in the previous 2 years.

Isolation and preparation of isolates. Isolations were made by placing lesioned leaf segments overnight in petri dishes lined with moistened filter paper, and then transferring cirrhi individually with a sterile needle to plates of yeast-malt agar (YMA) amended with streptomycin at $50 \mathrm{mg} / \mathrm{liter}$. The resulting monopycnidial isolates were stored at $4^{\circ} \mathrm{C}$ and transferred to fresh

TABLE 1. Alleles ${ }^{\mathrm{a}}$ at five restriction fragment length polymorphism (RFLP) loci in two Mycosphaerella graminicola strains inoculated on field plots of winter wheat in Corvallis, OR, in 1998, 1999, and 2000

\begin{tabular}{lcc}
\hline & \multicolumn{2}{c}{ Alleles } \\
\cline { 2 - 3 } RFLP locus $^{\text {b }}$ & Strain 15-4A.9 & Strain 2-2A.12 \\
\hline pSTL2 & 3 & 7 \\
pSTL10 & 3 & 3 \\
pSTL53 & 3 & 8 \\
pSTS192A & 7 & 6 \\
pSTS199 & 1 & 2 \\
\hline
\end{tabular}

a Alleles originally numbered by McDonald and Martinez (17) in order of decreasing frequency in the population.

b All genomic DNA digested with PstI. 
YMA every 3 to 5 months. Isolates were tracked by their leaf, row, replicate, cultivar, and year of origin.

DNA extraction, restriction digestion, and Southern blotting. Total DNA from each isolate was extracted using a cetyltrimethylammonium bromide (CTAB) protocol as modified by McDonald and Martinez (17). Eight micrograms of DNA from each isolate was digested with the restriction enzyme PstI. The DNA fragments were separated by electrophoresis through $1.0 \%$ agarose gels. The DNA fragments in the gels were transferred to Hybond N+ nylon membranes (Amersham Biosciences, Piscataway, $\mathrm{NJ}$ ) by vacuum blotting. The membranes were then baked at $80^{\circ} \mathrm{C}$ and stored until needed.

Five anonymous DNA probes (pSTL2, pSTL10, pSTL53, pSTS192, and pSTS199) originating from an M. graminicola genomic library $(15,17)$ were hybridized sequentially to the Southern blots. These probes hybridized to one locus each, with the exception of pSTS192, which hybridizes to two unlinked loci (18). The probe pSTS199 provided limited information, as the two inoculant isolates had, respectively, the first and second most common alleles at that locus, but the data were still included in the analysis. In addition, hybridization was performed with DNA fingerprint probe 9-A5a, supplied by S. Goodwin, which contains the coding sequence for a reverse transcriptase gene and is thought to be part of a transposable element (9). Hybridization with probe 9 -A5a produces multiple bands, with ample polymorphism among $M$. graminicola individuals.
The probes were radioactively labeled with $\mathrm{dCT}\left[{ }^{32} \mathrm{P}\right]$. The labeled probes were hybridized to the membranes overnight at $65^{\circ} \mathrm{C}$ in a hybridization incubator. The membranes were then washed at $55^{\circ} \mathrm{C}$ and exposed to PhosphorImager screens (Molecular Dynamics, Amersham BioSciences, Piscataway, NJ). After exposure, the radioactive probes were stripped off the membranes. Each gel included three lanes of a 1-kb DNA ladder for sizing of bands, one lane at each edge and a third in the center. Isolates were re-run if they could not be scored unambiguously.

Clone correction and isolate classification. Isolates were only retained in the samples if their multi-locus haplotype could be unambiguously determined at all loci, and their DNA fingerprint was clearly legible. Analyses of allelic diversity were conducted on the total sample, without clone correction. Multilocus haplotypes and DNA fingerprints were used to clone-correct (4) the isolate populations for analyses of genotypic diversity. Only one immigrant or recombinant with a given fingerprint was retained in the clone-corrected sample. Inoculant types were also arbitrarily removed from the sample during clone-correction if more than one isolate of the strain was obtained from the same sampling point in a plot, but not otherwise.

For all isolates in the 1998, 1999, and 2000 populations, DNA fingerprints were scored for the presence or absence of certain bands specific to each inoculant strain, and this information was added as "loci" to isolates' multilocus haplotypes. The following procedure was used to choose and score the fingerprint bands.

TABLE 2. Non-clone-corrected frequencies of restriction fragment length polymorphism (RFLP) alleles in a natural Mycosphaerella graminicola population sampled from noninoculated wheat plots in 1999 and 2000 in Corvallis, OR

\begin{tabular}{|c|c|c|c|c|c|c|c|c|c|c|}
\hline RFLP locus ${ }^{\mathrm{a}}$ & Allele ${ }^{b}$ & \multicolumn{3}{|r|}{ Year } & $\begin{array}{l}\text { Chi-squared statistic } \\
\text { for difference } \\
\text { between years }\end{array}$ & $\mathrm{df}$ & $P$ value & \multicolumn{3}{|c|}{$\begin{array}{l}\text { Other reported Oregon } \\
\text { allele frequencies }\end{array}$} \\
\hline \multirow{5}{*}{$\begin{array}{l}n=\text { sample size } \\
\text { pSTL2 }\end{array}$} & & 20 & 46 & 66 & & & & 711 & 56 & 43 \\
\hline & 2 & 0.300 & 0.457 & 0.409 & & & & & 0.429 & \\
\hline & 3 & 0.200 & 0.022 & 0.076 & & & & & 0.036 & \\
\hline & 7 & 0.050 & 0.000 & 0.015 & & & & & & \\
\hline & 8 & 0.000 & 0.022 & 0.014 & & & & & & \\
\hline \multirow{4}{*}{ pSTL10 } & 2 & 0.000 & 0.065 & 0.045 & & & & & 0.107 & 0.00 \\
\hline & 3 & 0.350 & 0.217 & 0.257 & & & & 0.21 & 0.357 & 0.29 \\
\hline & 9 & 0.000 & 0.022 & 0.015 & & & & & & \\
\hline & 27 & 0.000 & 0.043 & 0.030 & & & & & & \\
\hline \multirow[t]{5}{*}{ pSTL53 } & 1 & 0.550 & 0.348 & 0.409 & 15.8 & 9 & 0.071 & 0.50 & 0.536 & 0.46 \\
\hline & 2 & 0.050 & 0.196 & 0.152 & & & & 0.13 & 0.107 & 0.02 \\
\hline & 15 & 0.050 & 0.000 & 0.015 & & & & & & \\
\hline & 23 & 0.050 & 0.000 & 0.015 & & & & & & \\
\hline & 24 & 0.050 & 0.000 & 0.015 & & & & & & \\
\hline \multirow[t]{3}{*}{ pSTS192A } & 1 & 0.900 & 0.826 & 0.848 & 2.5 & 2 & 0.287 & 0.92 & 0.911 & 0.95 \\
\hline & 2 & 0.100 & 0.065 & 0.076 & & & & 0.04 & 0.054 & 0.05 \\
\hline & 3 & 0.000 & 0.109 & 0.076 & & & & & 0.000 & 0.00 \\
\hline \multirow[t]{8}{*}{ pSTS199 } & 1 & 0.500 & 0.196 & 0.288 & 8.9 & 8 & 0.351 & & 0.434 & \\
\hline & 2 & 0.400 & 0.565 & 0.515 & & & & & 0.415 & \\
\hline & 3 & 0.000 & 0.065 & 0.045 & & & & & 0.019 & \\
\hline & 5 & 0.100 & 0.065 & 0.076 & & & & & 0.038 & \\
\hline & 6 & 0.000 & 0.022 & 0.015 & & & & & & \\
\hline & 21 & 0.000 & 0.022 & 0.015 & & & & & & \\
\hline & 26 & 0.000 & 0.022 & 0.015 & & & & & & \\
\hline & 27 & 0.000 & 0.022 & 0.015 & & & & & & \\
\hline
\end{tabular}

\footnotetext{
a All genomic DNA restricted with PstI.

b Alleles originally numbered by McDonald and Martinez (17) in order of decreasing frequency in the population.

c All data reported on clone-corrected samples.

${ }^{\mathrm{d}}$ Zhan et al. (26).

e Chen et al. (3).

${ }^{f}$ Zhan et al. (25).
} 
Amplified fragment length polymorphism (AFLP)-Quantar software (Keygene N.V., Wageningen, The Netherlands) was used to estimate fragment lengths of all DNA fingerprint bands for all isolates. Bands were selected that consistently appeared in the fingerprints of one inoculant strain and never appeared in the fingerprints of the other inoculant strain. Six such bands were chosen: three from isolate 9 and three from isolate 12. For confirmation, bands estimated by AFLP-Quantar as within the ranges of the diagnostic bands of the inoculant strains were manually compared with those of inoculant-type isolates. Each such "locus" had one of two alleles, " 1 " or " 2 " (presence or absence).

Isolates were placed according to their multi-locus haplotype and their DNA fingerprint in one of the following categories: asexual progeny of isolate 9, asexual progeny of isolate 12, recombinant progeny of those strains (referred to hereafter as "912 recombinant"), recombinant progeny of an inoculant and an immigrant strain, or an immigrant. Two methods of classification were compared: a "manual" method and a Bayesian approach.

"Manual" classification. An isolate was classified as a 9-12 recombinant if its DNA fingerprint was composed of bands of each strain, as depicted by Zhan et al. (23). An isolate was classified as an inoculant-immigrant recombinant if it met the following criteria: (i) the isolate had alleles in common with the inoculant parent at two of the four loci pSTL2, pSTL10, pSTL53, and pSTS192; or (ii) in common with the inoculant parent, the isolate had at least one allele occurring in the natural population at a frequency of $<7 \%$; and (iii) the DNA fingerprint of the isolate shared $\geq 3$ bands with the inoculant parent, out of a mean of 10.8 bands in all fingerprints classified as recombinant. A threshold of three common bands was chosen because all six isolates in the 1999 to 2000 natural population that met the previous criterion (sharing one low-frequency allele with an inoculant) shared fewer than three bands of their DNA fingerprint with the relevant inoculant.

Bayesian assignment. The Bayesian clustering program Structure (19) was used to classify each isolate from an inoculated plot as either an inoculant clone, an immigrant, or the product of admixture (recombination) between inoculants or between an inoculant and an immigrant. Structure assumes that marker loci are unlinked and at linkage equilibrium with one another within populations, assumptions that are reasonable for these markers (17) and for M. graminicola (23), respectively. To each isolate, Structure assigns a posterior probability (conditional probability based on observed allele frequencies) that it is descended from each source population, such that it is possible to set a threshold for inferring descent from each particular source population, and to identify "admixture," or descent from multiple source populations.

Structure analyses were run with a burn-in length of 500,000 iterations followed by a run length of 1,000,000 iterations. Haplotypes consisted of alleles at the 11 loci described previously. The first analysis was conducted without prior population information, using the two inoculants and the natural population, and testing for potential population structure of between one and five populations. The best fit (i.e., the highest posterior probability) was obtained with $\mathrm{K}=3$ populations, corresponding to the two inoculant populations and the natural population. Then, isolates from the inoculated plots for each year, together with the 60 isolates from the natural population, were assigned to the three identified populations. A posterior probability of $\geq 10 \%$ was arbitrarily assumed to indicate descent from a particular population.

Statistical analysis. Isolates sampled from the natural population in four replicate plots of noninoculated 'Stephens' totaled 20 in 1999 and 46 in 2000 before clone correction, and 18 and 42 in the 2 years, respectively, after clone correction. To determine whether samples from the 2 years could be combined, variation in allelic proportions was subjected to a contingency chi-squared analysis as in Workman and Niswander (22), with appropriate modification for a haploid organism.

The combined natural population and the 1998, 1999, and 2000 populations from the inoculated plots were each analyzed separately after clone correction for genotypic diversity and linkage equilibrium using MultiLocus version 1.2 (Agapow and Burt, www.bio.ic.ac.uk/evolve/software/multilocus). Diversity and equilibrium statistics, and their $P$ values, were calculated based on 1,000 randomizations.

Chi-squared tests were conducted using SAS PROC FREQ (SAS Institute, Cary, NC) to compare the proportions of isolates classified as inoculant, recombinant, and immigrant in the three years.

\section{RESULTS}

Epidemics of different intensities developed in the 3 years (Fig. 1) due to varying weather conditions. In 1998, the epidemic developed consistently and strongly throughout the season. In 1999, a dry late spring held disease levels relatively low. In 2000, early disease levels were low due to below-average rainfall in March and April, but disease increased rapidly in May and June due to above-average May rainfall. Thus, final mean disease severities were higher in 1998 and 2000, and lower in 1999.

The natural populations sampled in 1999 and 2000 were found to have only one significant difference in allele frequencies by contingency chi-squared analysis (Table 2), and were considered as one population for the purpose of making comparisons to isolates from inoculated plots. Allele frequencies from both years, and the weighted mean frequencies of the two samples combined, are given in Table 2. The allele frequencies found in this population agree closely with those found for the Willamette Valley M. graminicola population in $1990(3,12,26)$ and 1995 (23) (Table 2). Chi-squared analysis was used to compare allele frequencies in the 1992 population described by Chen et al. (3) with those in our combined 1999 to 2000 natural population. No significant differences were found in allele frequencies at any of the five loci $(P \geq 0.10)$.

From the inoculated plots, alleles at single-copy RFLP loci and DNA fingerprints were determined for 132, 127, and 122 isolates in the 3 years, respectively. Frequencies of alleles that comprised $\geq 1 \%$ of the sample in at least one of the 3 years are reported in Table 3. Chi-squared analysis indicated there were significant differences in allele frequencies among the 3 years at all loci except pSTS199 (Table 3).

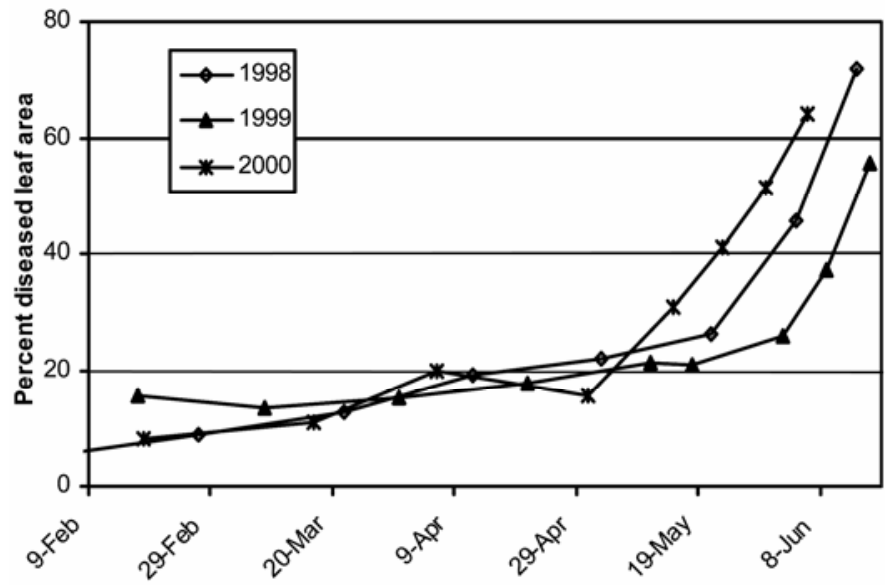

Fig. 1. Progress of Septoria tritici blotch in plots of winter wheat cultivar Stephens inoculated with Mycosphaerella graminicola spores early in each of three growing seasons (1997 to 1998, 1998 to 1999, and 1999 to 2000) in Corvallis, OR. Disease was assessed on a whole-canopy basis as percent leaf area covered by lesions. 
Multi-locus analysis of the clone-corrected 60-isolate 1999 to 2000 natural population showed 59 different genotypes (Table 4). The genotypic diversity statistic, which can be thought of as the probability that two individuals chosen at random would have different multilocus haplotypes, was 0.999 , and no departure from gametic equilibrium was indicated.
After clone-correction, the sample sizes from the inoculated plots were 107, 106, and 107 isolates, respectively, for the years 1998, 1999, and 2000 (Table 5). The numbers of isolates excluded by clone correction are shown in Table 5 (under "E"). Analyses of genotypic diversity were performed on the clone-corrected samples. Genotypic diversity in populations from the inoculated plots was

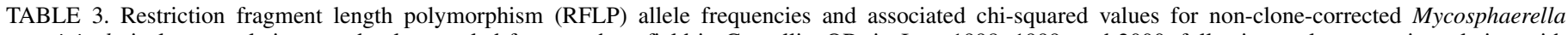

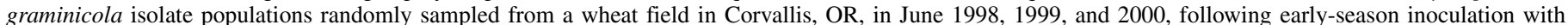
M. graminicola rare-allele strains

\begin{tabular}{|c|c|c|c|c|c|c|c|}
\hline \multirow[b]{2}{*}{ Locus $^{\mathrm{a}}$} & \multirow[b]{2}{*}{ Allele ${ }^{b}$} & \multicolumn{3}{|c|}{ Year of isolate collection ${ }^{\mathrm{c}}$} & \multirow[b]{2}{*}{ Chi-squared statistic ${ }^{d}$} & \multirow[b]{2}{*}{ df } & \multirow[b]{2}{*}{$P$ value } \\
\hline & & 1998 & 1999 & 2000 & & & \\
\hline \multirow[t]{5}{*}{ pSTL2 } & 1 & 0.265 & 0.524 & 0.448 & 47.5 & 6 & $<0.0001$ \\
\hline & 2 & 0.217 & 0.131 & 0.384 & & & \\
\hline & 3 & 0.235 & 0.164 & 0.118 & & & \\
\hline & 6 & 0.007 & 0.033 & 0.000 & & & \\
\hline & 7 & 0.276 & 0.149 & 0.050 & & & \\
\hline \multirow[t]{8}{*}{ pSTL10 } & 1 & 0.334 & 0.510 & 0.651 & 36.1 & 4 & $<0.0001$ \\
\hline & 2 & 0.025 & 0.034 & 0.041 & & & \\
\hline & 3 & 0.623 & 0.413 & 0.241 & & & \\
\hline & 4 & 0.000 & 0.007 & 0.018 & & & \\
\hline & 8 & 0.000 & 0.036 & 0.009 & & & \\
\hline & 9 & 0.000 & 0.000 & 0.016 & & & \\
\hline & 10 & 0.000 & 0.000 & 0.016 & & & \\
\hline & 28 & 0.012 & 0.000 & 0.000 & & & \\
\hline \multirow[t]{11}{*}{ pSTL53 } & 1 & 0.173 & 0.315 & 0.504 & 55.2 & 10 & $<0.0001$ \\
\hline & 2 & 0.070 & 0.062 & 0.152 & & & \\
\hline & 3 & 0.270 & 0.232 & 0.124 & & & \\
\hline & 5 & 0.018 & 0.015 & 0.030 & & & \\
\hline & 6 & 0.130 & 0.146 & 0.097 & & & \\
\hline & 7 & 0.013 & 0.019 & 0.008 & & & \\
\hline & 8 & 0.286 & 0.164 & 0.056 & & & \\
\hline & 9 & 0.008 & 0.028 & 0.000 & & & \\
\hline & 10 & 0.008 & 0.000 & 0.010 & & & \\
\hline & 14 & 0.012 & 0.000 & 0.010 & & & \\
\hline & 17 & 0.000 & 0.007 & 0.010 & & & \\
\hline \multirow[t]{8}{*}{ pSTS192A } & 1 & 0.416 & 0.567 & 0.745 & 74.4 & 6 & $<0.0001$ \\
\hline & 2 & 0.008 & 0.049 & 0.059 & & & \\
\hline & 3 & 0.031 & 0.125 & 0.080 & & & \\
\hline & 6 & 0.306 & 0.141 & 0.031 & & & \\
\hline & 7 & 0.226 & 0.117 & 0.016 & & & \\
\hline & 19 & 0.008 & 0.000 & 0.010 & & & \\
\hline & 22 & 0.000 & 0.000 & 0.010 & & & \\
\hline & 23 & 0.000 & 0.000 & 0.017 & & & \\
\hline \multirow[t]{7}{*}{ pSTS199 } & 1 & 0.384 & 0.379 & 0.287 & 5.9 & 4 & 0.2079 \\
\hline & 2 & 0.499 & 0.541 & 0.563 & & & \\
\hline & 3 & 0.020 & 0.000 & 0.026 & & & \\
\hline & 5 & 0.014 & 0.038 & 0.069 & & & \\
\hline & 6 & 0.049 & 0.035 & 0.009 & & & \\
\hline & 13 & 0.000 & 0.000 & 0.016 & & & \\
\hline & 16 & 0.012 & 0.000 & 0.000 & & & \\
\hline
\end{tabular}

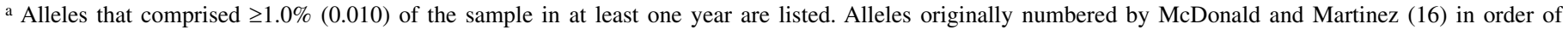
decreasing frequency in the population.

b All genomic DNA restricted with PstI.

${ }^{c}$ Frequencies were determined separately for samples from each of four replicate plots, and then averaged across the replicates.

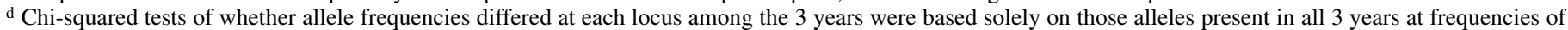
$\geq 1.0 \%$.

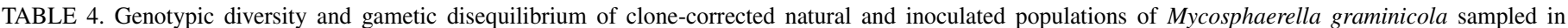
Corvallis, OR

\begin{tabular}{|c|c|c|c|c|c|c|}
\hline \multirow[b]{2}{*}{ Population } & \multirow[b]{2}{*}{ Sample size } & \multirow[b]{2}{*}{ Number of genotypes } & \multicolumn{2}{|c|}{ Genotypic diversity $^{a}$} & \multicolumn{2}{|c|}{ Gametic disequilibrium $^{\mathrm{b}}$} \\
\hline & & & Statistic & $P$ value & $\mathrm{I}_{\mathrm{A}}$ & $P$ value \\
\hline Natural population & 60 & 59 & 0.999 & 0.821 & -0.125 & 0.970 \\
\hline \multicolumn{7}{|l|}{ Inoculated populations } \\
\hline 1998 & 107 & 74 & 0.955 & $<0.001$ & 1.487 & $<0.001$ \\
\hline 1999 & 106 & 78 & 0.980 & $<0.001$ & 0.672 & $<0.001$ \\
\hline 2000 & 107 & 102 & 0.999 & 0.334 & 0.124 & 0.019 \\
\hline
\end{tabular}

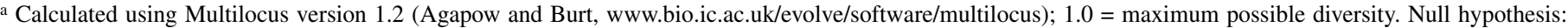
statistic does not differ from 1.0.

${ }^{\mathrm{b}} \mathrm{I}_{\mathrm{A}}=$ index of association $(0=$ no gametic disequilibrium $)$. Null hypothesis: $\mathrm{I}_{\mathrm{A}}=$ zero. 
lowest in 1998, intermediate in 1999, and similar to that in the natural population in 2000 (Table 4). Significant departures from gametic equilibrium in all samples from the inoculated plots (Table 4) were presumably due to the associations among loci of the inoculant progeny, both sexual and asexual.

Table 5 presents the classification of the sampled isolates as inoculant clones, recombinant, or immigrant. In the 3 years respectively, inoculants comprised 36,23 , and $1 \%$ of the total populations by manual classification, and 36,22 , and $2 \%$ by Structure analysis. Detected recombinants were 15,9 , and $13 \%$ of the total populations by manual classification, and 19,8 , and $16 \%$ by Structure analysis. The substantial drop in inoculants recovered in 2000 reflects the fact that inoculation was performed a month later in that year than in the previous 2 years. Using the Table 5 tallies by Structure, the ratio of inoculants to immigrants differed significantly among the 3 years $\left(\chi^{2}=40.3\right.$, df $\left.=2, P<0.0001\right)$, and also between 1998 and 1999, the years with comparable inoculation dates $\left(\chi^{2}=6.0, \mathrm{df}=1, P=0.01\right)$.

Isolation of the pathogen from the moderately resistant cultivar Foote was more difficult, and 'Foote' plots were not available for sampling in 1998, resulting in a small sample (Table 5). Though this small sample size precluded a meaningful statistical analysis, the distribution of these isolates among the inoculant, recombinant, and immigrant categories was consistent with results obtained with the more numerous Stephens' isolates (Table 5).

Despite the difference in epidemic intensities in 1998 and 1999 (Fig. 1), recombinants comprised similar percentages of all inoculant progeny (sexual and asexual) in the 2 years (Table 6). The high standard deviation for the 1999 estimate was due mainly to the absence of detected recombinants in the second of the four replicate plots. Using tallies by Structure, the ratio of recombinants to inoculants differed significantly among the 3 years $\left(\chi^{2}=\right.$ 24.3, df $=2, P<0.0001)$, but this was due to the lack of inoculants recovered in 2000 . The recombinant/inoculant ratio did not differ between 1998 and $1999(P=0.28)$, even though the 1999 epidemic was much less intense due to the dry spring. The ratio of 9-12 recombinants to inoculants also did not differ between 1998 and 1999 (Fisher's exact test, $P=0.24$ ).

\section{DISCUSSION}

In 1998 and 1999, the percentages of recombinants in the total end-of-season samples from the susceptible cultivar Stephens reflected the relative dominance of inoculant strains, with inoculants better established relative to immigrants in 1998 than in 1999. Presumably, the end-of-season share of immigrant-immigrant recombinants was higher in 1999 than in 1998, as immigrants constituted a larger proportion of the total sample in the second year, but our experimental protocol did not allow us to distinguish this fraction of the population. The detectable recombinant share of all inoculant progeny (inoculant clones plus recombinants) was similar in both years: 32 to $35 \%$. Our previous study of fruiting bodies on the same cultivar had found 93 and $32 \%$ of $M$. graminicola fruiting bodies were ascocarps in 1998 and 1999, respectively (5). Taken together, these findings are consistent with the hypothesis that sexual recombination makes a relatively consistent contribution to $M$. graminicola population structure, despite differences in epidemic severity and proportion of ascocarps. It should be noted that our results are based on the release and recapture of only one isolate of each mating type. Fortunately, there was approximately equal establishment of inoculants 9 and 12 in each of the 3 years. However, M. graminicola isolates vary in fitness (aggressiveness) (6), and a larger set of marked isolates could have generated different results.

TABLE 6. Mycosphaerella graminicola recombinants as a percentage of inoculant progeny ${ }^{\mathrm{a}}$ from a 3-year mark-release-recapture experiment in Corvallis, OR

\begin{tabular}{lccc}
\hline Replicate plot of Stephens wheat & 1998 & 1999 & 2000 \\
\hline 1 & $33 \%$ & $67 \%$ & $100 \%$ \\
2 & $41 \%$ & $0 \%$ & $100 \%$ \\
3 & $32 \%$ & $29 \%$ & $78 \%$ \\
4 & $33 \%$ & $31 \%$ & $100 \%$ \\
Mean & $35 \%$ & $32 \%$ & $95 \%$ \\
SD & $4.2 \%$ & $27.4 \%$ & $11.0 \%$ \\
\hline a Detected recombinants/(inoculants + detected recombinants) $\times 100$, using \\
Structure tallies from Table 5.
\end{tabular}

TABLE 5. Classification of clone-corrected populations of Mycosphaerella graminicola isolates collected in June 1998, 1999, and 2000 from field plots in Corvallis, OR, following early-season inoculation with M. graminicola rare-allele strains 15-4A.9 (inoculant 9) and 2-2A.12 (inoculant 12) ${ }^{\mathrm{a}}$

\begin{tabular}{|c|c|c|c|c|c|c|c|c|c|c|c|c|c|c|c|}
\hline \multirow{4}{*}{$\begin{array}{l}\text { Host and category } \\
\text { of isolate }\end{array}$} & \multicolumn{15}{|c|}{ Year of isolate collection } \\
\hline & \multicolumn{5}{|c|}{1998} & \multicolumn{5}{|c|}{1999} & \multicolumn{5}{|c|}{2000} \\
\hline & \multirow[b]{2}{*}{$\mathrm{E}^{\mathrm{c}}$} & \multicolumn{4}{|c|}{ Classification method } & \multicolumn{5}{|c|}{ Classification method } & \multirow[b]{2}{*}{$E^{c}$} & \multicolumn{4}{|c|}{ Classification method } \\
\hline & & No. & $\%$ & No. & $\%$ & $\mathrm{E}^{\mathrm{c}}$ & No. & $\%$ & No. & $\%$ & & No. & $\%$ & No. & $\%$ \\
\hline \multicolumn{16}{|l|}{ 'Stephens'd } \\
\hline Inoculant 9 & 11 & 18 & 17 & 17 & 17 & 4 & 11 & 10 & 11 & 10 & 0 & 0 & 0 & 0 & 0 \\
\hline $9-12$ recomb & & 7 & 6 & 7 & 7 & & 1 & 1 & 1 & 1 & & 2 & 2 & 2 & 2 \\
\hline 9-imm recomb & & 2 & 2 & 5 & 5 & & 5 & 4 & 3 & 3 & & 6 & 5 & 8 & 7 \\
\hline 12-imm recomb & & 7 & 7 & 6 & 6 & & 3 & 4 & 4 & 5 & & 7 & 6 & 8 & 8 \\
\hline 9-12-imm recomb & & 0 & 0 & 2 & 2 & & 0 & 0 & 0 & 0 & & 0 & 0 & 0 & 0 \\
\hline Total & & 107 & 100 & 107 & 100 & & 106 & 100 & 106 & 100 & & 107 & 100 & 107 & 100 \\
\hline \multicolumn{16}{|l|}{ 'Foote'd } \\
\hline Inoculants & & & & & & & 4 & $29 \%$ & & & & 0 & $0 \%$ & & \\
\hline
\end{tabular}


Although data from varied environments would be necessary for confirmation, our results suggest there may be a rough balance among the factors determining the recombinant percentage of $M$. graminicola descendants at the end of a growing season. Dry conditions reduce pycnidiospore spread and infection, and thus slow the rate of pseudothecial formation from the encounter of opposite mating-type individuals; however, pseudothecia that do form can spread their spores in the absence of rain better than can pycnidia (8), thus, by the end of the season increasing the proportion of infections caused by sexual descendents. Wet conditions increase epidemic development and lesion density through the rain-splashed transfer of pycnidiospores, and thereby increase the likelihood of opposite mating types meeting and producing pseudothecia. By the end of the season, this increases the fraction of fruiting bodies that are pseudothecia, but reduces the proportion of infections caused by sexual descendants.

To confirm that differences among years in end-of-season inoculant frequencies reflected differences in early-season establishment, it would have been necessary to sample from these plots soon after inoculation. This sampling was omitted due to resource limitations, as this was part of a larger experiment that addressed several other questions.

The manual and Structure methods of classifying isolates produced generally similar results. A detailed comparison of manual versus Structure assignments suggests that Structure's assignments may be more accurate overall, because the program computes probabilities based on the frequencies of observed alleles at all loci relative to reference population frequencies. Structure detected a slightly higher proportion of recombinants in 1998 and 2000 than were detected by visual inspection. However, the $10 \%$ threshold for inferring ancestry did lead to a false Structure identification. An isolate from 2000 was given 90.4 and $9.1 \%$ probability of having isolate 12 and immigrant ancestry, respectively, which classified it as an isolate-12 clone. Yet inspection of the fingerprint revealed several immigrant bands, and the isolate had an immigrant allele at one of the single-copy probe loci.

Structure detected two cases of "three-way" recombination among inoculant strains 9, 12, and an immigrant isolate, both in 1998. Binomial probabilities give us $95 \%$ confidence that, based on the pre-clone-correction sample size of 132 in 1998, three-way recombinants make up less than $2.2 \%$ of the end-of-season $M$. graminicola population. This suggests that the total time required to complete two sexual cycles and one asexual cycle generally exceeds the Oregon winter wheat growing season, in line with previous evidence (5). However, samples gathered from debris later in the summer might provide stronger support for the occurrence of two cycles of sexual reproduction per year.

Detection of recombination frequencies by release and recovery of tagged isolates depends on timely artificial inoculation, so that inoculant strains achieve substantial coverage relative to immigrants. The frequency of recombinants remained in the same range in 2000 as in 1998 and 1999, yet only one inoculant was detected in 2000. As Structure produced results similar to those of manual inspection, we do not believe that the tally of inoculantimmigrant isolates was inflated. Rather, it is likely that the low frequency of inoculants in 2000 was simply due to sampling error, as immigrants constituted $85 \%$ of the end-of-season population.

Would we have detected higher frequencies of recombinants by using more RFLP probes? The frequency of 9-12 recombinants would not have increased, because there were no cases in which the multi-locus haplotype was inoculant, yet the fingerprint indicated mixed ancestry. The frequency of isolates classified as inoculant-immigrant recombinants would likely have increased, because the presence in the multi-locus haplotype of rare alleles from the inoculant parents was the first indicator of a possible recombinant, and this would have been detected more often if data from more loci were available. However, the inclusion of additional loci from DNA fingerprint bands unique to each inoculant supplemented the single-copy probes and increased the power of our analysis.

The comparison of allele frequencies in natural $M$. graminicola populations in Table 2 indicates that frequencies remained fairly stable through the end of the 1990s, as had been found for the 1990 to 1995 period $(3,25)$. The high gene and genotype diversity of the 1999 to 2000 natural population, and its gametic equilibrium, were in line with all previous research $(12,23,26)$.

Taking the report by Cowger et al. (5) and the present study together, we see that even though intense epidemics may lead to greater ascocarp production, which in turn may enhance disease establishment the following year, recombinant frequencies may not be substantially higher in these populations than in those that develop during low-disease years. This is likely due to the concomitant acceleration of asexual reproduction under conditions favorable to disease. In any case, the recommended management practice of planting moderately resistant cultivars serves both to minimize disease progress within a season and reduce production of the ascocarps that initiate future epidemics.

\section{ACKNOWLEDGMENTS}

This research was supported in part by award 97-353034537 of the USDA National Research Initiative Competitive Grants Program. We thank J. P. Martinez, A. Preston, and especially L. Sayavedra Soto for crucial technical advice and support; M. Hoffer for work on the field experiment; and staff of the OSU Botany and Plant Pathology Field Laboratory for help with establishment and maintenance of the field plots. Helpful criticisms of the editor and reviewers substantially strengthened this report.

\section{LITERATURE CITED}

1. Beaumont, M. A., and Rannala, B. 2004. The Bayesian revolution in genetics. Nature 5:251-261.

2. Brown, J. K. M. 2000. Estimation of rates of recombination and migration in populations of plant pathogens. Phytopathology 90:320-323.

3. Chen, R.-S., Boeger, J. M., and McDonald, B. A. 1994. Genetic stability in a population of a plant pathogenic fungus over time. Mol. Ecol. 3:209218.

4. Chen, R.-S., and McDonald, B. A. 1996. Sexual reproduction plays a major role in the genetic structure of populations of the fungus Mycosphaerella graminicola. Genetics 142:1119-1127.

5. Cowger, C., McDonald, B. A., and Mundt, C. C. 2002. Frequency of sexual reproduction by Mycosphaerella graminicola on partially resistant wheat cultivars. Phytopathology 92:1175-1181.

6. Cowger, C., and Mundt, C. C. 2002. Aggressiveness of Mycosphaerella graminicola isolates from susceptible and partially resistant wheat cultivars. Phytopathology 92:624-630.

7. DiLeone, J. A., Coakley, S. M., Karow, R., and Mundt, C. C. 1997. Biology and control of Septoria diseases of winter wheat in western Oregon. Crop Science Research Report No., Oregon State University.

8. Eriksen, L., Shaw, M. W., and Ostergard, H. 2001. A model of the effect of pseudothecia on genetic recombination and epidemic development in populations of Mycosphaerella graminicola. Phytopathology 91:240-248.

9. Goodwin, S. B., Cavaletto, J. R., Waalwijk, C., and Kema, G. H. J. 2001. DNA fingerprint probe from Mycosphaerella graminicola identifies an active transposable element. Phytopathology 91:1181-1188.

10. Hunter, T., Coker, R. R., and Royle, D. J. 1999. The teleomorph stage, Mycosphaerella graminicola, in epidemics of septoria tritici blotch on winter wheat in the UK. Plant Pathol. 48:51-57.

11. Kema, G. H. J., Verstappen, E. C. P., Todorova, M., and Waalwijk, C. 1996. Successful crosses and molecular tetrad and progeny analysis demonstrate heterothallism in Mycosphaerella graminicola. Curr. Genet. 30:251-258.

12. Linde, C. C., Zhan, J., and McDonald, B. A. 2002. Population structure of Mycosphaerella graminicola: From lesions to continents. Phytopathology 92:946-955.

13. Mank, J. E., and Avise, J. C. 2004. Individual organisms as units of analysis: Bayesian-clustering alternatives in population genetics. Genet. Res. 84:135-143.

14. McDonald, B. A., and Linde, C. 2002. Pathogen population genetics, evolutionary potential, and durable resistance. Annu. Rev. Phytopathol. 40:349-379. 
15. McDonald, B. A., and Martinez, J. P. 1990. Restriction fragment length polymorphisms in Septoria tritici occur at a high frequency. Curr. Genet. 17:133-138.

16. McDonald, B. A., and Martinez, J. P. 1990. DNA restriction fragment length polymorphisms among Mycosphaerella graminicola (anamorph Septoria tritici) isolates collected from a single wheat field. Phytopathology 80:1368-1373.

17. McDonald, B. A., and Martinez, J. P. 1991. DNA fingerprinting of the plant pathogenic fungus Mycosphaerella graminicola (anamorph Septoria tritici). Exp. Mycol. 15:146-158.

18. McDonald, B. A., and Martinez, J. P. 1991. Chromosome length polymorphisms in a Septoria tritici population. Curr. Genet. 19:265-271.

19. Pritchard, J. K., Stephens, M., and Donnelly, P. 2000. Inference of population structure using multilocus genotype data. Genetics 155:945959.

20. Shaw, M. W., and Royle, D. J. 1989. Airborne inoculum as a major source of Septoria tritici (Mycosphaerella graminicola) infections in winter wheat crops in the UK. Plant Pathol. 38:35-43.

21. Shaw, M. W., and Royle, D. J. 1993. Factors determining the severity of epidemics of Mycosphaerella graminicola (Septoria tritici) on winter wheat in the UK. Plant Pathol. 42:882-899.

22. Workman, P. L., and Niswander, J. D. 1970. Population studies on Southwestern Indian tribes. II. Local genetic differentiation in the Papago. Am. J. Hum. Genet. 22:24-49.

23. Zhan, J., Mundt, C. C., and McDonald, B. A. 1998. Measuring immigration and sexual reproduction in field populations of Mycosphaerella graminicola. Phytopathology 88:1330-1337.

24. Zhan, J., Mundt, C. C., and McDonald, B. A. 2000. Estimation of rates of recombination and migration in populations of plant pathogens - A reply. Phytopathology 90:324-326.

25. Zhan, J., Mundt, C. C., and McDonald, B. A. 2001. Using restriction fragment length polymorphisms to assess temporal variation and estimate the number of ascospores that initiate epidemics in field populations of Mycosphaerella graminicola. Phytopathology 91:1011-1017.

26. Zhan, J., Pettway, R. E., and McDonald, B. A. 2003. The global genetic structure of the wheat pathogen Mycosphaerella graminicola is characterized by high nuclear diversity, low mitochondrial diversity, regular recombination, and gene flow. Fungal Genet. Biol. 38:286-297. 